\title{
Rébellions urbaines et déviances policières
}

Approche configurationnelle des relations entre les “jeunes" des Minguettes et la police (1981-1983)

Urban rebellions and police brutality. A configurational approach of relationships between youngsters of the Minguettes and police (1981-1983)

\section{Abdellali Hajjat}

\section{(Q) OpenEdition}

Journals

\section{Édition électronique}

URL : http://journals.openedition.org/conflits/18839

DOI : $10.4000 /$ conflits. 18839

ISSN : $1777-5345$

Éditeur :

CCLS - Centre d'études sur les conflits lilberté et sécurité, L'Harmattan

Édition imprimée

Date de publication : 8 juillet 2014

Pagination : 11-34

ISBN : 978-2-343-04155-1

ISSN : 1157-996X

\section{Référence électronique}

Abdellali Hajjat, «Rébellions urbaines et déviances policières », Cultures \& Conflits [En ligne], 93 |

printemps 2014, mis en ligne le 02 juillet 2015, consulté le 30 mars 2021. URL : http://

journals.openedition.org/conflits/18839; DOI : https://doi.org/10.4000/conflits.18839 


\title{
Rébellions urbaines et déviances policières
}

\author{
Approche configurationnelle des relations entre les "jeunes" \\ des Minguettes et la police (1981-1983)
}

\section{Abdellali HAJJAT}

Abdellali Hajjat est Maître de conférences en science politique à l'Université Paris Onest Nanterre La Défense (Groupe d'analyse politique) et membre associé $d u$ Centre Maurice Halbwachs, équipe "Enquêtes, Terrains, Théories ». Il est diplômé de l'IEP de Lyon et docteur en sociologie (EHESS). Abdellali a récement publié La Marche pour l'égalité et contre le racisme aux éditions Amsterdam (2013). Il est aussi l'auteur, avec Marwan Mohammed, de Islamophobie. Comment les élites françaises fabriquent le problème musulman (La Découverte, 2013).

$\mathrm{D}$ ans quelles conditions la légitimité de l'usage de la force physique par la police peut-elle être directement remise en cause par les gouvernés ? 1 L'objectif de cet article est d'analyser une forme particulière de délégitimation, la rébellion urbaine ${ }^{2}$, qui est considérée par les forces de l'ordre comme une des menaces les plus importantes à leur propre exercice de la violence physique. La question est de savoir quand et comment l'autorité policière est contestée, et quelles sont les logiques sociales et les stratégies d'action qui déterminent le recours à la rébellion, entendue au sens le plus strict comme « l'opposition individuelle ou collective à l'exécution d'un ordre, qu'il s'agisse d'une saisie judiciaire, d'une perquisition, d'une arrestation, etc. ${ }^{3}$ ». Dans

1. Je remercie Laurent Bonelli, Marwan Mohammed, Cédric Moreau de Bellaing, Pierre Gilbert et les deux évaluateurs/trices anonymes pour leur précieuse relecture de ce texte, dont les imperfections relèvent de ma seule responsabilité. Cet article présente quelques résultats d'un travail de recherche collectif, mené dans le cadre du programme ANR «Genrebellion » (2006-2011), dirigé par Michelle Zancarini-Fournel et Sophie Béroud.

2. On envisage les phénomènes émeutiers de la région lyonnaise comme des formes de rébellion urbaine dans la mesure où l'on distingue le répertoire d'action (usage collectif et illégitime de la violence physique) du contexte dans lequel ils s'inscrivent (opposition à un ordre légal). Autrement dit, on préfère utiliser le terme de « rébellion » plutôt que celui d' «émeute " parce que l'une n'est pas réductible à l'autre. La rébellion peut bien sûr s'exprimer par d'autres modes d'action que l'émeute, et l'émeute peut se réaliser dans un tout autre contexte que celui de la rébellion (cas des émeutes « rituelles» de la Saint-Sylvestre en France). Voir ZancariniFournel M., "Généalogie des rébellions urbaines en temps de crise (1971-1981) », Vingtième siècle. Revue d'bistoire, 84, 2004, pp. 119-127. 
cette perspective, la rébellion urbaine peut être analysée comme un micro-événement révélateur d'une « crise du consentement 4 » à la domination exercée par les forces de police. L'idée est de rendre compte d'une situation particulière où la croyance en la légitimité de l'action policière se dissipe, se fissure, remettant en cause le "minimum de volonté d'obéir 5 », fondement central à la stabilité de la domination légale-rationnelle ${ }^{6}$.

Pour analyser la contestation de l'autorité policière par la rébellion, nous proposons de revenir sur les origines des rébellions des Minguettes (Vénissieux, Rhône) de 1981 et 1983, en mobilisant le concept éliasien de " configuration » 7 . Une analyse " configurationnelle » de la rébellion nous semble pertinente dans la mesure où elle constitue une alternative crédible à la théorie de la «frustration relative », souvent mobilisée, implicitement ou explicitement, pour expliquer les rébellions de l'automne 2005 en France. Selon cette perspective, "les émeutes urbaines du mois de novembre 2005 ont révélé certaines limites d'un modèle d'intégration à vocation universaliste, fondé sur une communauté de citoyens égaux formellement mais soumis à des traitements inégalitaires dans la réalité 8 ». Ainsi, "l'une des causes majeures de la frustration et du ressentiment de cette jeunesse française [réside dans la] disjonction entre ce qu'implique le “modèle républicain d'intégration” en termes d'égalité des chances, de méritocratie, de droits et de citoyenneté, et la situation réelle de stigmatisation, de ségrégation et de discrimination reposant sur leur précarité sociale, celle de leurs parents, et sur leur origine “immigrée” 9 ». Dans le sillage du modèle de Ted Gurr ${ }^{10}$, le principal ressort de la

3. Nicolas J., La rébellion française. Mouvements populaires et conscience sociale, 1661-1789, Paris, Gallimard, 2008, p. 30.

4. Gobille B., "Mai 68, crise du consentement et rupture d'allégeance ", in Damamme D., Gobille B., Matonti F. et Pudal B. (eds.), Mai Juin 68, Paris, Éditions de l’Atelier, 2008, pp. 15-31.

5. Weber M., Économie et société, Paris, Presses-Pocket, vol. 1, 1995 [1921], p. 285.

6. La domination légale-rationnelle est une « forme de domination légitime revêtant un caractère rationnel et reposant sur la croyance en la légalité des règlements arrêtés et au droit de donner des directions qu'ont ceux qui sont appelés à exercer la domination par ces moyens » (ibid., p. 289).

7. Norbert Élias entend par configuration « la figure toujours changeante que forment les joueurs; elle inclut non seulement leur intellect, mais toute leur personne, les actions et les réactions réciproques. (...) Cette configuration forme un ensemble de tensions. L'interdépendance des joueurs, condition nécessaire à l'existence d'une configuration spécifique est une interdépendance en tant qu'alliés, mais aussi en tant qu'adversaires. Le concept de configuration s'applique aussi bien aux groupes relativement restreints qu'aux sociétés formées par des milliers ou des millions d'êtres interdépendants. Qu'il s'agisse de professeurs et d'élèves dans une classe, de médecins et de patients dans un groupe thérapeutique (...), tous forment ensemble des configurations relativement transparentes; mais les habitants d'un village, d'une grande ville, ou d'une nation forment, eux aussi, des configurations, bien que celles-ci ne soient pas directement perceptibles du fait de la plus grande longueur et différenciation des chaînes d'interdépendance qui y relient les hommes. À partir de là, on peut essayer de saisir indirectement les particularités de ces configurations complexes par l'analyse des chaînes d'interdépendance " (Élias N., Qu'est-ce que la sociologie ?, La Tour d'Aigues, Éd. de l'Aube, 1991, pp. 157 et $s q$.$) .$

8. Lagrange H. et Oberti M., "Introduction. Intégration, ségrégation et justice sociale », in Émentes urbaines et protestations, une singularité française, Paris, Presses de Sciences Po, 2006, p. 33. 
rébellion résiderait dans une frustration, produite par le décalage entre les attentes - qui seraient suscitées par le « modèle républicain d'intégration français » - et l'absence de satisfaction de celles-ci (chômage, précarité, racisme, etc.).

Or, ce modèle explicatif est largement remis en cause par la sociologie de l'action collective parce que le chemin du mécontentement à la révolte est loin d'être mécanique. Il existe en effet quantité de situations où l'accumulation de frustrations ne se traduit pas par la révolte ${ }^{11}$. Et, « si le modèle de la frustration relative est aujourd'hui largement abandonné 12 » pour analyser les mobilisations collectives, notamment parce que le passage de la privation relative à la mobilisation n'est pas clairement explicité, pourquoi en serait-il autrement pour les rébellions urbaines ? Il s'avère plus intéressant de se pencher sur la dynamique de la rébellion et de s'attacher au comment plutôt qu'au pourquoi. Autrement dit, il s'agit de poser les questions suivantes : comment la croyance en la légitimité de l'activité policière est-elle remise en cause par la rébellion ? Comment est délégitimé ce qui paraissait jusque-là légitime ? C’est en ce sens que l'analyse configurationnelle se révèle être intéressante. Dans la mesure où elle prend au sérieux les relations d'interdépendance, les rapports de force et l'équilibre des tensions sociales, elle permet de penser les moments de rupture de consentement. On fait ainsi l'hypothèse que la croyance en la légitimité de l'activité policière dépend d'un certain équilibre des tensions et, à l'inverse, que la remise en cause de cette croyance est le produit d'un déséquilibre des rapports de force. L'enjeu consiste donc à savoir selon quelles logiques sociales et politiques se produit ce déséquilibre. En mobilisant le concept de configuration, il ne s'agit pas d'opposer mais au contraire d'articuler la logique structurelle et la logique conjoncturelle pour comprendre la rébellion ${ }^{13}$.

9. Ibid., p. 26. On retrouve cette idée plus implicitement chez d'autres auteurs. Voir par exemple Mucchielli L., "Introduction. Les émeutes de novembre 2005 : les raisons de la colère ", in Mucchielli L. et Le Goaziou V. (eds.), Quand les banlienes brûlent... Retour sur les émentes de novembre 2005, Paris, La Découverte, 2006, pp. 20-30; Kokoreff M., Sociologie des émentes, Paris, Payot, 2008, pp. 22-23.

10. Gurr T., Why Men Rebel ?, Princeton, Princeton University Press, 1970.

11. Dans son analyse des rébellions de 2005, Lagrange reconnaît que dans $31 \%$ des cas, il ne se produit pas d'émeute alors que les « conditions " (territoire, Zones Franches Urbaines, chômage, taille des famille, indice de ségrégation, etc.) sont réunies et que, dans $19 \%$ des cas, il y a émeute alors que les « conditions » ne sont pas réunies... (Lagrange $\mathrm{H}$., « La structure et l'accident ", in Lagrange H. et Oberti M., Émeutes..., op. cit., p. 115).

12. Fillieule O., «Émergence et développements des mobilisations », in Cohen A., Lacroix B. et Riutort Ph. (eds.), Nouveau manuel de science politique, Paris, La Découverte, coll. « Grands repères ", p. 520.

13. Sur le rapport entre structure et conjoncture, voir Mauger G., « Les raisons de la colère. Sur l'émeute de novembre 2005 », in Béroud S., Gobille B., Hajjat A. et Zancarini-Fournel M. (eds.), Engagements..., op. cit., pp. 25-41. La plupart des travaux ont insisté sur le rôle de la conjoncture et sur la relation dialectique entre le local et le national, mais la plupart tendent à privilégier une approche structurelle en raison d'un éloignement du terrain pendant les rébellions de 2005. L'enquête par le bas de Marwan Mohammed est une des rares à parvenir à articuler le rapport conjoncture/structure (Mohammed M., «Bandes de jeunes, émeutes et politisations ", in La formation des bandes. Entre la famille, l'école et la rue, Paris, PUF, 2011, pp. 382-402). 
Par ailleurs, la contestation de l'autorité policière pose la question du choix du répertoire d'action ${ }^{14}$ de la rébellion. Il faut distinguer deux modalités générales avec, d'un côté, l'usage de la violence physique sous toutes ses formes possibles (violence contre l'intégrité physique des policiers, destruction de mobiliers urbains, incendie de véhicules, etc.) et, de l'autre, l'usage des répertoires d'action légitimes de l'action collective (pétition, manifestation, sit-in, grève de la faim, etc.). Dans les discours de qualification ou de disqualification, ces deux formes de rébellion sont généralement opposées et contradictoires : la première est considérée comme illégitime, "a-politique ", «proto-politique » ou « infra-politique » ${ }^{15}$, tandis que la seconde est considérée comme légitime, moderne et politique. Il nous semble que cette opposition devrait être discutée ${ }^{16}$ et l'on peut contester l'affirmation selon laquelle « les émeutes (...) renvoient (...) à des formes d'exclusion qui interdisent à une souffrance, à une inégalité ou à une injustice de se conflictualiser, de prendre des formes d'expression politiques 17 ». Au lieu de postuler une déconnexion totale ou, à l'inverse, une connexion nécessaire entre émeute et expression politique, nous voulons poser la question du passage de l'usage illégitime de la violence physique à l'usage d'un répertoire d'action légitime ${ }^{18}$. Là encore, une perspective configurationnelle nous semble pertinente parce qu'elle permet de prendre au sérieux les marges de manœuvre à la disposition des acteurs pour faire basculer le jeu en leur faveur. Il ne s'agit pas d'adopter la théorie du « choix rationnel » (calcul des coûts et profits d'une action) mais d'envisager la confrontation des jeunes avec la police dans « une dynamique de l'escalade maîtrisée de part et d'autre, au terme de laquelle la violence physique, lorsqu'elle est employée, est le plus souvent ajustée au niveau de violence ou de menace auquel on fait face 19 ». Grâce aux archives disponibles, il est possible de mettre en lumière les données fondamentales de la configuration et d'essayer de comprendre les choix stratégiques des acteurs, notamment les jeunes rebelles dans leur confrontation avec les forces de police.

14. Tilly C., La France conteste de 1600 à nos jours, Paris, Fayard, 1986.

15. Mauger G. et Fossé-Poliak C., «La politique des bandes », Politix, n¹4, 1991, pp. 27-43; Mauger G., L'émente de novembre 2005 : une révolte protopolitique, Bellecombe-en-Bauges, Éd. du Croquant, 2006.

16. Pour une discussion de cette opposition, voir Hajjat A., « À la frontière du politique. Action et discours des “jeunes de cité" de SOS Avenir Minguettes (1981-1983) ", in Béroud S., Gobille B., Hajjat A. et Zancarini-Fournel M. (eds.), Engagements, rébellions et genre dans les quartiers populaires en Europe (1968-2005), Paris, Éditions des archives contemporaines, 2011, pp. 13-24 ; Mohammed M., "Bandes de jeunes, émeutes et politisations », op. cit.

17. Lagrange H. et Oberti M., «Introduction... », op. cit., p. 23. C’est moi qui souligne.

18. Une socio-histoire des mobilisations contre les « violences policières" reste encore à écrire, mais quelques études de cas et analyses d'acteurs ont été publiées : Jobard F., Bavures policières? La force publique et ses usages, Paris, La Découverte, 2002 ; dossier « Dammarie-lèsLys : les militants de l'incertitude », Vacarme, 21, automne 2002 ; " Du Comité national contre la double peine au Mouvement de l'immigration et des banlieues. Entretien avec Tarik Kawtari ", in Boubeker A. et Hajjat A. (eds.), Histoire politique des immigrations (post)coloniales. France 1920-2008, Paris, Éditions Amsterdam, 2008, pp. 207-214 ; Kokoreff M., La force des quartiers : de la délinquance à l'engagement politique, Paris, Payot, 2003 et Sociologie..., op. cit., pp. 241-270.

19. Jobard F., "Sociologie politique de la "racaille" ", in Lagrange H. et Oberti M. (eds.), Émentes urbaines.., op. cit., p. 69. 
Enfin, il s'agit de mettre en lumière les enjeux d'une des principales pommes de discorde entre l'autorité policière et les rebelles : la reconnaissance des déviances policières, notamment l'usage « abusif » de la force physique 20. En effet, la politique de sanction des instances de contrôle des forces de police peut prêter le flanc à l'accusation d'« impunité » dans la mesure où « les dénonciations d'usage abusif de la force par la police aboutissent moins souvent à une sanction que celles de tout autre type d'atteintes; et, lorsque ces cas de violences sont sanctionnés, ils le sont proportionnellement moins gravement que les autres formes de déviances policières 21 ». Il s'est ainsi développé chez une partie de la population un sentiment d'injustice qui est au cœur de la contestation de l'autorité policière et dont il faut saisir les conditions de production au travers des interactions entre police et population.

Cet article propose une analyse de la configuration locale pour expliquer les conditions de possibilités de la rébellion. À partir du cas des rébellions des Minguettes de 1981 et 1983 22, il vise à étudier trois questions imbriquées : la rébellion comme forme de contestation de l'autorité policière, les conditions du passage à un répertoire d'action légitime par les rebelles, et la question de la reconnaissance des déviances policières violentes comme élément central dans les conditions de possibilité de la rébellion. Les historiens et les sociologues des quartiers populaires s'accordent à dire que les rébellions des Minguettes sont les symboles de la «crise des banlieues » 23 . Elles ont fait l'objet de nombreuses analyses, s'appuyant surtout sur le discours médiatique, mais on sait finalement peu de choses des acteurs de l'époque, en particulier sur les rebelles eux-mêmes. Cette lacune est d'autant plus problématique que, parmi les rebelles de 1981 et 1983, on en retrouve certains à l'origine de la Marche pour l'égalité et contre le racisme d'octobre-décembre 1983, événement fondateur de toute une génération de militants immigrés et des quartiers populaires ${ }^{24}$. On espère donc participer au renouvellement de l'analyse de ces événements grâce à la consultation d'archives inédites et à la réalisation d'entretiens avec des acteurs-clés des événements, et à la confrontation des récits et points de vue de la police et des habitants du quartier ${ }^{25}$.

20. Voir Jobard F., Bavures..., op. cit., 2002 ; Montjardet D., Ce que fait la police, Paris, La Découverte, 1996.

21. Moreau de Bellaing C., "Violences illégitimes et publicité de l'action policière ", Politix, 873, 2009, p. 126.

22. Même si nous évoquons la rébellion de 1981, notre attention se porte essentiellement sur celle de 1983 parce que les sources archivistiques sont plus abondantes et parce que cette dernière permet d'envisager les trois dimensions du problème présentées en introduction.

23. Voir, entre autres, Bachmann C. et Le Guennec N., Violences urbaines. Ascension et chute des classes moyennes à travers cinquante ans de politique de la ville, Paris, Hachette Littératures, 2002 [1996], pp. 331 et sq. Plus récemment : Jobard F., "An overview of French riots, 19812004", in Waddington D., Jobard, F. et King M., Rioting in the UK and France : a comparative analysis, Cullompton, Devon/Portland, Or., Willan Pub, 2009, pp. 27-38.

24. Beaud S. et Masclet O., "Des "marcheurs" de 1983 aux "émeutiers" de 2005. Deux générations sociales d'enfants d'immigrés ", Annales. Histoire, Sciences Sociales, 4, 2006, pp. 809843 ; Hajjat A., «La Marche pour l'égalité et contre le racisme », in Pigenet M. et Tartakowski M. (eds.), Histoire des mouvements socianx, France, 19e-21e siècles, Paris, La Découverte, 2012. 


\section{Contester l'autorité policière : la rébellion de mars 1983}

Pour comprendre l'usage de la violence physique par des habitants des Minguettes au début des années 1980, il est certes important de souligner les " causes de la colère ", c'est-à-dire les facteurs sociaux tels que la concentration de la pauvreté, la déstructuration de la classe ouvrière, le chômage massif, la ségrégation spatiale, le racisme, etc. ${ }^{26}$ Cependant, il peut être intéressant de l'analyser aussi en l'inscrivant dans une configuration sociale et politique ainsi que dans les rapports de forces qui structurent le quartier, en particulier entre les jeunes et la police, en faisant l'hypothèse que les rébellions des Minguettes s'expliquent en grande partie par la rupture d'un certain équilibre, fragile et précaire, dans les relations entre jeunes et police.

Cet équilibre repose jusqu'au début des années 1980 sur l'existence de deux formes d'institution sociale. D'un côté, s'est constitué un espace relativement autonome où les jeunes, parfois formés en bandes, peuvent se rencontrer, manger et discuter entre eux à l'abri du regard extérieur (famille, corps enseignant, éducateurs, police). De l'autre, il existe un corps policier dont l'action contre la délinquance est légitimée et acceptée par les habitants du quartier tant qu'elle n'est pas perçue comme une attaque à leur dignité ou à leur honneur social. On se gardera donc d'employer le terme polémique de «zone de non-droit » dans la mesure où, aux Minguettes, l'action de la police est généralement acceptée et rarement empêchée.

Tous les témoignages recueillis et les rapports de police montrent que, de la construction des premières tours en 1967 jusqu'à la fin des années 1970, il existe un faible niveau de violence entre habitants. Il est bien sûr difficile de se faire une idée précise de la réalité de la délinquance dans la mesure où les chiffres de la police sont une construction statistique qui révèle moins l'état « réel » de la délinquance que la (non)précision du processus de comptabilisation et/ou l'intensité de l'activité répressive. De plus, il n'est pas possible de

25. Cet article mobilise une petite partie d'un matériau diversifié. Il s'agit d'archives publiques (nationales [politique de la ville et ministère de l'Intérieur], départementales [versement du cabinet du Préfet de 1968 à 1986], municipales de Vénissieux), d'archives privées (CGT, Parti socialiste, CIMADE, militants comme Salika Amara, Toumi Djaïdja, Farid Taalba, etc.), de la presse locale et nationale (dossiers constitués à la bibliothèque municipale de la Part Dieu à Lyon de 1979 à 1985) et d'entretiens avec une vingtaine d'acteurs et actrices des Minguettes, de la Marche, des groupes Zaama de banlieue et de SOS Avenir Minguettes, ainsi que des responsables religieux et des fonctionnaires. Dans la mesure où les entretiens sont hautement subjectifs et ont été réalisés après les faits (près de trente ans), il est inévitable que les interviewés reconstruisent le passé en fonction de leur position présente. Les citations d'entretiens ne sont donc utilisées dans cet article que pour fournir un éclairage subjectif et qu'à partir du moment où elles contiennent des informations croisées avec des sources archivistiques ou des travaux scientifiques existants.

26. Voir notamment Dubet F., La galère : jeunes en survie, Paris, Fayard, 1987 ; Dubet F. et Lapeyronnie D., Quartiers d'exil, Paris, Seuil, 1992 ; Beaud S. et Pialoux M., Violences urbaines, violence sociale : genèse des nouvelles classes dangereuses, Paris, Fayard, 2003 ; Mucchielli L. et Le Goaziou (eds.), Quand les banlienes..., op. cit. ; Kokoreff M., Sociologie..., op. cit. 
connaître les chiffres spécifiques à la ville de Vénissieux car les statistiques disponibles englobent les faits de délinquance constatés dans l'ensemble du département du Rhône. On peut néanmoins discerner quelques caractéristiques générales de la délinquance vénissiane grâce aux informations fragmentaires des rapports de la police judiciaire. Alors que la population rhodanienne s'accroît de 4,5\% entre 1974 et 1980, on constate durant cette période une croissance de 58,2 \% du nombre de crimes et délits constatés dans le département (de 55104 à 87 148) sachant que celle-ci résulte principalement de l'augmentation des faits de "délinquance » (de 44882 à 72 529) - la " grande criminalité » et la «criminalité moyenne » passant respectivement de 741 à 1439 et de 9481 à 1318027.

Mais cette croissance globale ne se répartit pas équitablement entre les cinq circonscriptions distinguées par la police judiciaire (voir figure 1) : ville de Lyon (centre), Villefranche (nord), Tarare (ouest), Givors-Grigny (sud) et Est-lyonnais (Bron, Saint-Priest, Saint-Fons, Décines, Vaulx-en-Velin, Vénissieux, Villeurbanne). En effet, on constate des variations importantes entre ces zones du département en termes de taux de délinquance (nombre de faits de délinquance pour 1000 habitants). Entre 1976 et 1980 28, le taux de délinquance départemental passe de 31,72 à 49,04 \%o mais on distingue deux types de situations : les deux circonscriptions au-dessus de la moyenne départementale (Lyon et Villefranche) et les trois autres situées en-dessous (Givors, Est-lyonnais, Tarare). Ainsi, le taux de délinquance dans l'Est-lyonnais est inférieur et croît moins vite que ceux de la ville de Lyon et de la zone de Villefranche. Entre 1976 et 1980, le taux est-lyonnais passe de 14,84 à 27,5 \%o tandis qu'à Lyon il croît de 44,16 à 62,15 \%o et à Givors-Grigny de 24,56 à 31,18 \%o. Il faut attendre l'année 1981 pour que le taux délinquance de la zone est-lyonnaise dépasse celui la zone de Givors-Grigny, sachant que les deux zones ont un profil économique et sociologique comparable à cette période (emploi industriel, composition sociale à majorité ouvrière, taux de chômage élevé, etc.).

27. Afin de pouvoir réaliser des comparaisons géographique et temporelle, nous nous basons sur la distinction policière opérée entre "grande criminalité », "criminalité moyenne » et « délinquance », qui sont des catégories regroupant de multiples délits classés selon leur « degré de gravité ». Celles-ci sont évidemment les produits de luttes de définition de la réalité sociale et devraient être discutées, mais nous ne disposons pas d'éléments empiriques sur la construction de ces catégories pour pouvoir le faire.

28. La comparaison entre les cinq circonscriptions ne peut se faire qu'entre 1976 et 1981 parce que la distinction entre " grande criminalité », "criminalité moyenne » et " délinquance " n’apparaît qu'en 1976 pour la ville de Lyon et, à partir de 1982, la nomenclature des crimes et délits change au profit des deux catégories centrales d'" atteintes aux personnes » et d'« atteintes aux biens ». Les données statistiques de la circonscription «Est-lyonnais » n'apparaissent pas en tant que telles dans les rapports de la police judiciaire : elles sont donc déduites par soustraction des données disponibles pour l'ensemble du département et les quatre autres circonscriptions. 


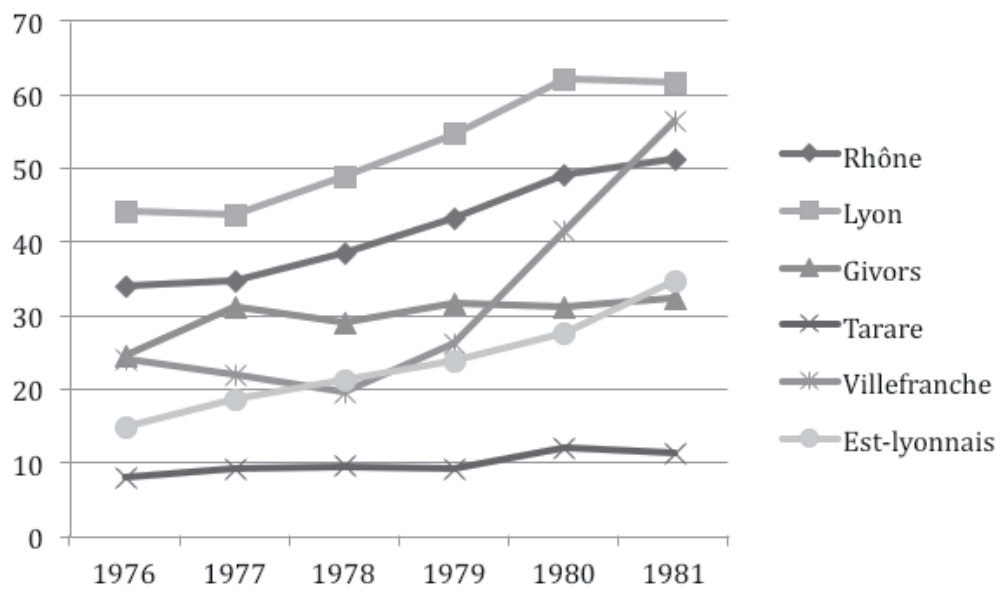

Figure 1 : Taux de délinquance dans le Rhône entre 1976 et 1981 (en \%o).

Source : Ministère de l'Intérieur (Direction centrale de la police judiciaire), Aspects de la criminalité en France d'après les statistiques de la police judiciaire, Paris, La Documentation française, 1976-1981.

Avant le début des années 1980, les différents acteurs sociaux et politiques de Vénissieux font bien le constat de la multiplication des faits de délinquance aux Minguettes, mais cette réalité n'est pas (encore) leur principale préoccupation. À l'occasion du conseil municipal extraordinaire du 29 octobre 1976, organisé sous un chapiteau installé au milieu de la ZUP, les premières doléances des acteurs locaux portent essentiellement sur le chômage des jeunes, l'enseignement, le sport, l'immigration, le logement et les loyers. La question de la délinquance en tant que telle est reléguée, dans le compte-rendu du conseil municipal publié dans le journal Vénissieux Infos, aux « quelques points qui n’ont pas pu être abordés » : "S’il est vrai que la délinquance des jeunes pose un problème, elle ne reste que le fait d'une infime minorité. La jeunesse dans son ensemble est sérieuse et travailleuse mais quelles perspectives lui offrent ceux qui nous gouvernent ? 29 ».

L'idée d'un phénomène marginal est vérifiée par les chiffres du commissaire de Vénissieux/Saint-Fons qui identifie, en septembre 1980, seulement vingt-deux «familles connues défavorablement » des services de police et quarante-trois « délinquants multirécidivistes " dans la commune de Vénissieux ${ }^{30}$. Parmi ces derniers, on retrouve seulement quatre filles ; dix-huit sont mineurs, vingt et un entre 18 et 30 ans et quatre de plus de 30 ans ; vingttrois ont la nationalité française (dont deux Français de naissance, vingt d'origine algérienne et un d'origine espagnole), vingt sont étrangers (seize Algériens, trois Tunisiens et un Espagnol). Il s'agit surtout de petite délinquance dans la mesure où ils sont connus des services de police essentiellement

29. Archives municipales de Vénissieux (AMV), 226W172. Vénissieux Infos, octobre 1976, p. 8.

30. Archives départementales du Rhône (ADR), 2230W09. Liste des « délinquants habituels » de Vénissieux dressée par le commissaire de Vénissieux/Saint-Fons pour le commissaire central agglomération lyonnaise, 8 septembre 1980. 
pour des vols à l'étalage, de cyclomoteurs, parfois par effraction et pour « outrage et rébellion ».

Ainsi, la délinquance est considérée comme le fait d'une « infime minorité » et n'est pas encore construite en problème public que les autorités politiques et policières doivent régler en priorité. En ce sens, on peut parler d'un équilibre, fragile et précaire, dans les relations sociales aux Minguettes. Bien que des tensions sociales entre les habitants apparaissent dans l'Est-lyonnais dès le début des années 1970 31, il s'est construit aux Minguettes une sorte de modus vivendi où la qualité des relations sociales est encore relativement épargnée par les divisions internes aux classes populaires produites par la montée du chômage et de la précarité sociale, même si celles-ci se font de plus en plus présentes. Comme le souligne une enquête sociologique menée dans le quartier en 1979-1980, il existe un "véritable mythe de l'insécurité (...) qui va très au delà des faits 32 ». En ce sens, « [1] es relations sociales [aux Minguettes] sont, dans l'ensemble, bonnes ou acceptables; par contre, la coexistence de ménages appartenant à des groupes ethniques ou sociaux trop différents est parfois difficile ou, au moins, conflictuelle ; ce n'est pas seulement la cohabitation Français/immigrés, mais aussi - et d'abord - celle de la fraction la plus aisée et la plus moderniste de la classe ouvrière (ouvriers qualifiés, employés, techniciens, grosso modo) et des fractions plus traditionnelles - françaises aussi bien qu'immigrés 33 ». Ainsi, la fracture entre fractions marginalisées et fractions intégrées des classes populaires se creuse peu à peu, favorisant l'identification et l'appropriation du territoire des Minguettes par les jeunes du quartier.

En effet, les jeunes qui appartiennent aux fractions les moins intégrées du monde ouvrier s'approprient certains espaces urbains dont une des fonctions consiste à les soustraire du regard des différentes formes d'autorité (familiale, éducative, scolaire et policière). Ils se retrouvent notamment dans la cafétéria Casino du centre commercial Vénissy, dans le snack «L’Ami Burger » et, pour ce qui concerne la bande de jeunes de Monmousseau (sous-quartier de la ZUP des Minguettes), dans le local mis à disposition par le bailleur social Logirel au pied de la tour $\mathrm{n}^{\circ} 10$ du quartier éponyme. Cette configuration favorise l'émergence de «bandes », peu structurées et constituées d'amis du même quartier, qui se distinguent des «blousons noirs » ${ }^{34}$ par leur origine sociale et leur lieu d'habitation. L'espace vénissian de la petite délinquance est marqué par les figures d'Amar Djaïdja (Monmousseau), de Frédéric Henry (boulevard Lénine) et, selon plusieurs acteurs du quartier, ces deux figures

31. Zancarini-Fournel M., "Généalogie... », art. cit.

32. Chevallier M., Pré-dossier Habitat Vie Sociale des Minguettes à Vénissieux, Lyon, Groupe de sociologie urbaine, juin 1980, p. X. Cette enquête a été réalisée dans le cadre du programme national « HVS » et pour le compte de l'Agence d'urbanisme de la Communauté urbaine de Lyon.

33. Ibid., p. III.

34. Mohammed M. et Mucchielli L. (eds.), Les bandes de jeunes, des « Blousons noirs " à nos jours, Paris, La Découverte, coll. « Recherches », 2007. 
bénéficient du soutien d'une partie de la population des Minguettes qui les aide occasionnellement, d'une manière ou d'une autre, à fuir la police. Petit à petit, une sorte de mythe populaire, a fortiori imaginaire et parfois exagéré, s'est construit : celui d'un Amar Djaidja « Robin des bois » ou «bandit social $35 »$ qui vole aux riches pour distribuer aux pauvres.

« [Amar Djaidja] avait même un côté Robin des Bois pour le quartier. Je me souviens une fois où il avait cambriolé je ne sais plus quel magasin de nourriture, un magasin du type Casino. Et le lendemain dans l'immeuble, [...] [quand] quasiment l'ensemble des habitants [...] ouvrait leur porte, il y avait devant leur porte un paquet de café, un paquet, une bouteille d'huile [petit rire]. Il avait aussi ces côtéslà. Je me souviens qu'à une époque, il avait fait la distribution de survêtements à tous les gamins de son quartier [...]. Mais bon, ça restait quand même de la délinquance et on va dire qu'il était un peu aux portes $[\ldots]$ du banditisme. (...) Il n'a pas été un vrai bandit mais en même temps... 36 »

Or, à partir du début des années 1980, l'équilibre de la configuration est progressivement bouleversé par deux facteurs, provoquant une montée de la tension sociale. Le premier réside dans le fait qu'un des moyens les plus efficaces à la disposition de la police pour lutter contre la délinquance et obtenir des renseignements est la menace d'expulsion des étrangers, qui représentent environ la moitié des « délinquants multirécidivistes » identifiés en juin 1980. Or, après la grève de la faim contre les expulsions menée par Christian Delorme, Jean Costil et Hamid Boukhrouma en avril 1981, le gouvernement socialiste nouvellement élu décide de suspendre les expulsions des jeunes étrangers (mai-juin 1981) ${ }^{37}$. Par conséquent, la police de Vénissieux se sent en quelque sorte «trahie » par le gouvernement et, lors d'une entrevue avec le maire communiste de Vénissieux Marcel Houël le 1er juillet 1981 (avant la rébellion de fin juillet), le préfet de police Jean Chevanche exprime le ressentiment de ses troupes : «La suspension des expulsions ne peut que rendre plus difficile l'action des services de police ; aucune menace ne pèse sur les malfaiteurs étrangers. On assiste d'ailleurs à un retour massif des délinquants expulsés 38 ».

Le second facteur tient au fait que la police ait l'impression, réelle ou supposée, que le territoire des Minguettes n'est plus sous contrôle, non pas parce

35. Hobsbawm E. J., Les bandits, Paris, La Découverte/Zones, 2008 [1969], pp. 28-42.

36. Entretien avec Djamel, membre de SOS Avenir Minguettes, Vénissieux, 18 novembre 2008. Ce point de vue est corroboré par de multiples articles du Progrès de Lyon de 1981-1983, qui participe à la création du mythe du « Prince des Minguettes ».

37. Mathieu L., La double peine. Histoire d'une lutte inachevée, Paris, La Dispute, 2006, pp. 83117.

38. ADR 2230W09. Compte-rendu de l'audience accordée par Jean Chevanche, préfet délégué pour la police, à Marcel Houël, maire de Vénissieux, le 1er juillet 1981. 
que le niveau de délinquance explose, mais parce que certains suspects réussissent à lui échapper. C'est le cas par exemple d'Amar Djaïdja qui réussit à s'échapper des mains des forces de l'ordre le 30 novembre 1981. Après avoir été interpellé pour le braquage manqué d'un magasin Intermarché de PierreBénite, cinq policiers de la Sûreté urbaine de Lyon se rendent avec lui aux Minguettes pour perquisitionner son domicile familial et interpeller ses complices présumés. Il parvient à s'échapper grâce à l'aide des habitants du quartier, comme le relate Le Progrès :

« Laissant le malfaiteur à la garde de deux d'entre eux, les trois autres fonctionnaires pénétraient dans l'immeuble. Mais leur présence dans ce quartier "chaud” de l'est lyonnais n'était pas (...) passée inaperçue. Aussitôt des petits groupes de quelques individus se formaient à proximité des voitures. Des petits groupes qui se fondirent rapidement en un seul d'une vingtaine de personnes lorsque retentirent les cris d'un des garçons appréhendés. (...) Les voitures de police étaient alors prises d'assaut, secouées et les policiers insultés. Dans la confusion, une des portières s'ouvrait et Djaïdja, menottes aux poings, en profita pour disparaître. Couvert par les acteurs de cette scène, il courait jusqu'à une voiture qui démarrait aussitôt. L'oiseau s'était envolé. ${ }^{39}$ »

Tout se passe comme si la suspension des expulsions et le sentiment de perte de contrôle territorial avaient bouleversé l'équilibre des rapports de force entre les jeunes, la petite délinquance et la police. On peut alors considérer que ce déséquilibre débouche sur une présence policière accrue et sur la succession d'incidents mineurs qui peuvent dégénérer, comme durant les rébellions de l'été 1981 et de mars 1983. Comme le souligne Toumi Djaïdja, habitant de Monmousseau et petit frère de Amar, la dégradation du bâti et des relations sociales s'est opérée assez brutalement, provoquant un cycle de montée en tension entre les jeunes et la police:

«Dans les années 1980, il y avait comme une forme de dégradation qui commençait à s'opérer dans le quartier... Une dégradation physique, matérielle. (...) Les tensions sont arrivées à une vitesse ! Il y a eu un revirement, un souffle de l'enfer sur les Minguettes. Ça a basculé dans le côté obscur. (...) Et la police a commencé à s’imposer davantage, se faire remarquer... ${ }^{40}$ »

De ce point de vue, il est intéressant de revenir sur la chronologie précise de la seconde rébellion (21 mars 1983), qui est à l'origine de l'organisation

39. Raffin R., «Un malfaiteur s'échappe à la faveur d'un début d'émeute », Le Progrès, $1^{\text {er }}$ décembre 1981.

40. Entretien avec Toumi Djaïdja, président de SOS Avenir Minguettes, Lyon, 11 décembre 2009. Un des indicateurs de la dégradation des relations sociales est le dépeuplement massif 
d'une grève de la faim par les jeunes du quartier, de la création de l'association SOS Avenir Minguettes et, plus tard, de la Marche pour l'égalité. Selon un rapport des Renseignements généraux, « un état comparatif de la délinquance entre les premiers trimestres des années 1982 et 1983 dans l'agglomération lyonnaise fait apparaitre une stagnation de la délinquance globale (+0,01\%) [et] ce phénomène général se répercute dans l'ensemble des communes de l'Est lyonnais ${ }^{41}$ ». Cependant, les événements du 21 mars 1983 «ont démontré (...) la tension qui régnait dans ces quartiers et qui risquait de dégénérer violemment au moindre incident entre les forces de l'ordre et jeunes immigrés 42 ».

En effet, le 21 mars à $8 \mathrm{~h}$, « le commissariat de Vénissieux procède à une perquisition dans [le local des jeunes de la] tour $n^{\circ} 10$ de l'avenue Gaston Monmousseau, où, selon des renseignements, des cambrioleurs avaient déposé le butin d'un cambriolage d'un magasin de fourrures commis au cours du week-end à Chambéry ${ }^{43}$ ». Compte tenu des incidents ayant eu lieu les jours précédents (contrôle d'identité ayant mal tourné, jets de bouteilles contre la police, etc.), un fourgon de gardiens de la paix est placé en renfort à proximité du local puis, après avoir été la cible de projectiles, une section d'intervention se rend au bas de la tour. Mais une autre descente de police est organisée au même moment, sans coordination avec la précédente. Cette présence accrue des forces de l'ordre produit sur les jeunes de Monmousseau l'effet d'être "attaqués ", comme le souligne le préfet de police Bernard Grasset : " Deux descentes de police au même endroit pour deux affaires différentes : (...) une affaire qui était une affaire de délinquance traditionnelle, l'autre qui était je sais plus quoi. Voilà les jeunes [qui] croient qu'ils sont attaqués ${ }^{44}$ ».

Après une perquisition fructueuse, les enquêteurs sortant du local et la section d'intervention en couverture devant la porte d'entrée sont la cible de jets de pierre et de barres de fer. Une première charge permet aux policiers de se dégager mais les rebelles se rassemblent près de la sortie du quartier et visent les véhicules quittant les lieux. Il aura fallu un tir nourri de grenades lacrymogènes et une nouvelle charge pour que l'ensemble des forces de police

des Minguettes. Entre 1975 et 1982, la population a chuté de $30 \%$, de 36000 à 25 300. Voir Chevallier M., Étude des stratégies résidentielles des ménages dans la ZUP des Minguettes, rapport ARCADES pour la ville de Vénissieux, septembre 1985.

41. ADR 1858W01. Renseignements généraux (RG), «Agitation parmi les jeunes immigrés dans l'est lyonnais et perspectives ", 2 mai 1983, p. 1.5390 faits ont été constatés au premier trimestre 1982 dans l'Est lyonnais et 5388 au premier trimestre 1983, sachant que la délinquance dans l'Est lyonnais représente environ $35 \%$ de la délinquance globale enregistrée dans l'agglomération lyonnaise. Les incendies de voitures suite à un « rodéo ", nombreux durant la rébellion de juillet 1981, accusent une baisse de $39 \%$.

42. Ibid., p. 2.

43. ADR 2519W18. Télégramme de la Direction départementale de la police urbaine (DDPU), "Affrontements entre forces de police et habitants de la ZUP des Minguettes à Vénissieux ", 21 mars 1983.

44. Entretien avec Bernard Grasset, préfet de police de Lyon entre le 16 juillet 1981 et le 17 juillet 1984, Rochefort, 22 février 2009. 
puisse sortir du quartier. Au total, douze personnes parmi les forces de l'ordre ont été blessées, dont le préfet de police Grasset et le directeur départemental de la police urbaine, un gardien de la paix a été hospitalisé (luxation de l'épaule) et une douzaine de véhicules de police ont été endommagés. Ces éléments de contexte, provenant des rapports de police, sont confirmés par un des jeunes habitants de la tour $n^{\circ} 10$ :

« Il y a eu la descente de police et après ça a commencé à partir en bagarre générale. D’autres unités policières sont arrivées et d'autres gens, venus d'autres quartiers sont arrivés, puis c'est monté en mayonnaise. La sauce est montée très vite et, en fin de matinée, on s'est retrouvé dans une bataille rangée. Il y avait des unités de différents corps policiers, des unités de gardes mobiles, des lacrymogènes. Je me suis retrouvé en bas de la Démocratie [nom du quartier] avec un flic qui avait un flingue, braqué sur moi. Il avait ramassé un coup de bâton par une mère de famille. Parce que ça impliquait non seulement les jeunes, mais aussi nos parents. Ma propre mère, ce jour-là, avait ramassé une grosse baffe par une femme flic dans l'ascenseur. (...) Heureusement que (...) le préfet de l'époque avait quand même du sang-froid (...) parce qu'il a débarqué, il était là. (...) Vu la situation, je pense que s'il avait donné l'ordre de rentrer dans le tas, il y aurait eu énormément de dégâts. Je pense que c'est un préfet qui a mesuré le danger, qui a mesuré où ça pouvait aller et qui avait fait le choix de faire le retrait, pour apaiser. 45 »

Ainsi, la contestation de l'autorité policière par les habitants de Monmousseau le 21 mars 1983 s'inscrit dans le cadre d'une configuration globale où les conditions de l'équilibre des tensions sociales ont peu à peu été bouleversées par le sentiment de perte de contrôle territorial et la suspension des expulsions, qui débouchent sur le renforcement de l'activité policière perçue comme une forme d'atteinte à la dignité sociale des habitants. Compte tenu de ce sentiment d'humiliation collective, l'équilibre qui s'était établi est rompu et, pour les habitants, ne mérite plus d'être maintenu : la croyance en la légitimité de l'action policière se fissure.

\section{Désarmer la police symboliquement}

Cependant, les conditions de possibilité de la contestation de l'autorité policière ne présument pas de la modalité de la rébellion. Dans cette perspective, la rébellion de mars 1983 est assez exceptionnelle dans la mesure où d'anciens émeutiers décident d'adopter l'action non-violente. En effet, « après le retrait des forces de police de la cité, un cortège de 350 jeunes habitants de la 
cité s'est constitué et s'est approché du commissariat de Vénissieux où il a été arrêté par un cordon de police. Après négociation [avec la municipalité], une réunion a été décidée pour les jours à venir et les manifestants se sont dispersés à 13 h15 46 ». Le cortège s'est transformé en sit-in devant la mairie (située juste à côté du commissariat) et une des revendications porte sur la libération d'un jeune des Minguettes, Antonio Bafunta alias « Tunch », arrêté pendant la rébellion par les forces de l'ordre. Par la suite, les jeunes de Monmousseau s'engagent, avec le soutien de militants d'extrême-gauche et/ou pro-immigrés, dans une grève de la faim (28 mars - 7 avril) et créent l'association SOS Avenir Minguettes (27 avril).

Pourquoi les jeunes ont-ils recours à la manifestation, au sit-in et à la grève de la faim et ne continuent-ils pas l'action émeutière ? Selon la vision dépréciative des Renseignements généraux, "après les affrontements du 21 mars, leur "image de marque" tant auprès de la presse écrite et parlée que de la population et des élus locaux s'était considérablement dégradée [et c]'est par simple opportunisme et pour redonner crédit à leur cause qu'une alternative non violente a été adoptée pour soutenir leur action 47 ». Cette disqualification des jeunes ne rendant pas compte de la complexité de la situation, il faut mettre en lumière plusieurs éléments pour comprendre le recours au répertoire d'action légitime par les rebelles.

Il faut tout d'abord souligner le fait que la pratique du répertoire d'action militant, notamment l'organisation d'un «comité de soutien » et la grève de la faim, n'est pas quelque chose d'inconnu pour les jeunes. Le quartier des Minguettes n'est en effet pas un « désert politique » avant 1983 parce qu'y habitent des militants actifs. Il s'agit notamment de militants comme Hamana Khira, Sif Guerdi, Toufik Kabouya (qui s’engagent à la fin des années 1970 dans le collectif informel Zaâma d'Banlieue ${ }^{48}$ ), d'un maoïste établi à l'usine Câbles de Lyon (Michel Ganozzi) et de femmes immigrées politisées (Dalila Mahamdi), qui jouent un rôle déterminant dans l'organisation de la grève de la faim. Ainsi, des comités de soutien à des jeunes incarcérés sont mis en place avant 1983 par ces habitants politisés du quartier : affaire Sélim Guéchi et Nordine Hammar (interpellés et condamnés à deux ans de prison en 1981) et affaire Gilles Cascarino (interpellé et condamné pour deux hold-up qu'il nie avoir commis en 1982) ${ }^{49}$. Ces affaires sont l'occasion pour les jeunes des Minguettes de connaître l'existence de pratiques militantes telles que les réunions, la recherche de soutiens, etc., surtout pour les petits frères des habitants

46. ADR 2519W18. Télégramme de la DDPU, op. cit.

47. ADR 1858W01. RG, «Agitation... », op. cit., p. 2.

48. Voir Nasri F., «Zaâma d’Banlieue (1979-1984) : les pérégrinations d'un collectif féminin au sein des luttes de l'immigration », in Béroud S., Gobille B., Hajjat A. et Zancarini-Fournel M. (eds.), Engagements..., op. cit., pp. 65-78.

49. Sur ces deux affaires, voir Nasri F., Les générations associatives des jeunes issus de l'immigration maghrébine : le cas de l'agglomération lyonnaise, thèse de science politique, sous la direction de Catherine Withol de Wenden, Sciences Po Paris, 2013. 
politisés (exemples de Djamel Mahamdi et de Mohamed Khira, membres de SOS Avenir Minguettes). Par ailleurs, la pratique de la grève de la faim, héritée des prisonniers politiques algériens pendant la guerre d'indépendance, se «banalise » à partir des années 196050 et est couramment utilisée en prison pour demander de meilleures conditions de détention ou protester contre l'expulsion des double-peine ${ }^{51}$. La grève de la faim fait donc partie de leur univers des possibles.

Néanmoins, le recours à la grève de la faim n'est pas évident puisque, selon le président de SOS Avenir Minguettes, « [le choix de faire une grève de la faim] n'était pas unanime. Mais [pour] une bonne partie de ceux qui devaient prendre d'une certaine façon la décision du choix des actions à mener... il s'est avéré que c'était (...) la voie de la sagesse 52 ». Parmi les jeunes ayant le plus pesé dans la décision, on retrouve Toumi Djaïdja qui réussit à convertir le capital social associé à son nom (et accumulé par son frère Amar) en capital politique ${ }^{53}$. Du point de vue de Toumi Djaïdja et de « ceux qui devaient prendre la décision ", l'usage de la violence physique était considéré comme une impasse :

«En réalité, ils nous collaient à la peau l'idée qu'on était voyous, violents, en marge de la société (...). C'était déjà partir avec ces handicaps... [Si on] rajoute encore de la violence, d'une façon ou d'une autre, elle se retourne contre nous. Le rapport de force était défavorable, c'était évident. La presse commençait à monter en épingle, juste avant la grève de la faim, que les fondements mêmes de la République étaient peut-être en danger, que la société française était en danger par le biais de ces banlieues qui sont en marge de la société... (...) Ils avaient monté, (...) vendu le truc tellement bien que, en réalité, il n'y avait pas d'autre alternative que de procéder de la sorte, c'est-à-dire de la non-violence. C'était même impératif à mon avis parce qu'on partait au casse-pipe ${ }^{54}$. »

La potentialité de l'action non-violente s'est donc actualisée en raison de la prépondérance des partisans de ce mode d'action et d'une analyse pragmatique de la configuration ${ }^{55}$ où les jeunes de cités, la police, les médias, les asso-

50. Siméant J., La grève de la faim, Paris, Presses de Science Po, 2009, pp. 19-23.

51. Lors de la grève de la faim contre la double-peine d'avril 1981, plusieurs détenus des prisons lyonnaises, dont Mohamed Khira (gréviste de la faim de 1983), s'engagent dans le comité de soutien en diffusant des tracts (ADR 2511W33. Note du cabinet du préfet, "Diffusion de tract dans les prisons de Lyon », 22 avril 1981). Je remercie Foued Nasri de m’avoir communiqué cette archive.

52. Entretien avec Toumi Djaïjja, Lyon, 18 décembre 2009.

53. Sur ce point, voir Hajjat A., "À la frontière du politique... », op. cit.

54. Ibid.

55. Ce constat pragmatique suppose un effort de distanciation et une capacité d'analyse de la situation, comme l'illustre le récit d'Edgar Poe sur la chute d'un pêcheur dans le Maelström, utilisé par Élias : «En bref : le pêcheur s’était vu impliqué dans un processus critique qui sem- 
ciations de soutien aux immigrés et le champ politique, à l'échelle locale et nationale, sont liés par des relations d'interdépendance. Ainsi, la stratégie nonviolente implique des répertoires d'action jugés plus « efficaces » que l'action émeutière pour lutter contre les déviances policières violentes. Ces ex-émeutiers arrivent en effet à la conclusion que le rapport de forces avec la police locale leur est défavorable dans le cadre d'une bataille de rue. Par le recours aux modes d'action légitimes, ils font d'une pierre deux coups : non seulement ils « court-circuitent » les moyens dont dispose la police pour emporter la partie (l'arrestation, la garde-à-vue, la poursuite judiciaire, voire l'emprisonnement), mais ils renversent aussi l'image stigmatisante du « jeune délinquant violent ». Autrement dit, le recours à l'action non-violente, qui est fondé sur le constat pragmatique de l'inégalité du rapport de force, permet de renverser le stigmate du délinquant violent et participe à « désarmer la police » symboliquement.

Le basculement de l'action émeutière à l'action politique légitime est aussi rendu possible par l'alliance opérée avec les militants chrétiens et non-violents par Christian Delorme, arrivé sur les lieux dès le 21 mars 1983, et Jean Costil, tous deux permanents de la Cimade. La question de l'influence de Delorme sur les jeunes de SOS Avenir Minguettes a fait couler beaucoup d'encre, que ce soit pour louer sa supposée capacité à les détourner de la violence, ou pour disqualifier les jeunes « ouailles » qui se seraient détournées de leur radicalité au profit d'un discours « œecuménique ». On a soit loué sa capacité de "pacification ", soit dénoncé sa responsabilité dans l'absence de « radicalisation ». Cette dernière critique est formulée par une partie du naissant mouvement autonome des «jeunes immigrés », en particulier par les militantes lyonnaises de Zaâma d'Banlieue qui accusent SOS Avenir Minguettes d'être « manipulés par les curés ».

Sans parler de pacification ou de récupération, il semble plus pertinent de parler de convergence stratégique. Si le choix de basculer de l'action émeutière à l'action politique légitime s'explique par la conscience d'un rapport de force inégal avec les forces de police, le sens de l'action non-violente est relativement différent pour ces hommes d'Église. En effet, pour ces militants chrétiens, la non-violence relève non seulement d'une stratégie politique mais aussi d'un principe éthique et religieux puisque, de leur point de vue, la grève de la

\footnotetext{
blait tout d'abord se soustraire totalement à son contrôle. Il se peut qu'il se soit cramponné un certain temps à de quelconques espoirs imaginaires. Des fantasmes de miracle, d'être invisibles venant à son secours lui ont peut-être traversé l'esprit. Après quelque temps, cependant, il retrouva son calme. Il commença à réfléchir avec davantage de sang-froid, et, prenant du recul, contrôlant sa peur, se regardant lui-même, pour ainsi dire, à distance, comme un homme qui formerait avec d'autres et avec les forces naturelles déchaînées une certaine constellation, il parvint à écarter ses pensées de lui-même et à les diriger sur la situation dans laquelle il était enfermée. Il reconnut alors, dans ce processus incontrôlable, les éléments qu'il pouvait utiliser en vue d'en contrôler les conditions de déroulement au mieux des intérêts de sa propre survie " (Engagement et distanciation. Contributions à la sociologie de la connaissance, Paris, Fayard, 1993, p. 76, c'est moi qui souligne).
} 
faim revêt une dimension sacrificielle et purificatrice ; dimension qui était étrangère aux habitudes des jeunes des Minguettes... Ainsi, ces jeunes et ces militants chrétiens convergent bien dans l'action non-violente, mais en ayant emprunté des chemins tout à fait différents. Et, du point de vue des grévistes de la faim, cette stratégie se révèle efficace dans la mesure où elle permet de « renvoyer la balle » à la police qui est sommée de reconnaître l'existence de déviances policières violentes.

\section{Des policiers « couverts » par la hiérarchie ?}

La reconnaissance des déviances policières est en effet le principal enjeu de cette forme de contestation de l'autorité policière. De ce point de vue, l'attitude du préfet de police Grasset ${ }^{56}$ par rapport aux rebellions des Minguettes est assez particulière. Dans le déroulement de la rébellion de mars 1983, la stratégie de Grasset est déterminante pour contenir la rébellion et éviter l'escalade de la violence alors qu'il est lui-même blessé en recevant un pavé au visage... Il mène une politique d'apaisement afin de diminuer la tension aux Minguettes, quitte à se mettre à dos la presse régionale et le corps policier, qui lui reprochent d'avoir été «faible » et d'avoir livré le territoire des Minguettes aux « voyous » 57 .

On peut dès lors souligner le caractère décisif d'un facteur, pas le seul bien sûr, qui favorise ou non, à court-terme, l'escalade de la violence physique pouvant se traduire par une extension géographique de la rébellion et la perpétuation de la contestation de l'autorité policière. Ce facteur est la perception, par les habitants, $d u$ degré d'union ou de division des différentes composantes de l'État - à savoir les différentes forces de police (municipale, nationale, gendarmerie, CRS, etc.), les différents niveaux d'autorités politiques (gouvernement, préfecture, mairie, etc.) et la justice pénale - quant à l'attitude à adopter face aux déviances policières violentes et à la rébellion. Cette attitude renvoie, d'un côté, au degré de «solidarité étatique » entre les représentants de l'État et les policiers mis en cause pour des conduites déviantes violentes et, de l'autre, au mode de règlement du conflit avec les rebelles. L’idée de solidarité étatique n'occulte pas le fait que tous les acteurs étatiques mentionnés ne constituent pas un groupe monolithique : il existe bien sûr des tensions et des contradictions au sein et entre la police, la justice et les autorités politiques.

56. Bernard Grasset (1933-) est le fils d'un instituteur socialiste et résistant de la CharenteMaritime, assassiné par la milice en 1944. Il est diplômé de l'Institut d'études politiques de Paris et entame sa carrière à la « préfectorale » en 1959 en tant que chef de cabinet du préfet des Ardennes. Après avoir occupé des postes de sous-préfet ou de secrétaire général de plusieurs départements métropolitains (Haute-Marne, Basses-Alpes, etc.) et d'outre-mer (Réunion), il fait son entrée dans plusieurs cabinets ministériels à partir de l'élection de Valéry Giscard d'Estaing (conseiller technique du Secrétaire d'État aux Départements d'Outre-Mer en 1974, chef de cabinet du Secrétaire d'État aux Postes et aux Télécommunications en 19771980). Il devient ensuite préfet des Hautes-Alpes (décembre 1980 - janvier 1981) et préfet délégué à la police du Rhône (16 juillet 1981 - 26 juillet 1984).

57. Voir Le Progrès, 22 mars 1983. 
Mais la perception de la solidarité étatique est au cœur du sentiment d'injustice des habitants des Minguettes qui dénoncent l'«impunité » des policiers déviants et des individus soupçonnés de crimes racistes. Ce sentiment est partagé par toute une génération d'individus socialisés dans la même communauté d'expérience ${ }^{58}$.

\begin{abstract}
"Cette génération se disait: "mais attends, tu as vécu ma vie, j’ai vécu la tienne ou quoi pour penser comme moi ?”. Quand tu parles avec des gens, [tu te rends compte qu']ils sont dans le même état d'esprit (...). C'était un sentiment partagé [selon lequel] la justice ne s'appliquait pas. Ils tuaient des gens. Je me souviens d'Ahmed Bouteldja tué en 1982. Sa maman était venue me voir et m'avait raconté l'histoire. Je me suis dit: "mais attends, ce n'est pas possible. On ne tue pas les gens comme ça”. Mais c'était normal, la justice couvrait ces crimes... Il y avait une impunité qu'on ne pouvait pas imaginer. On avait des jeunes de banlieue qu'on amenait dos contre le mur (...). On en faisait des voyous. Certains sont peut-être encore en prison. Alors qu'en face, il y avait de la soi-disant "légitime défense" et ils tuaient des gens. Ils étaient libérés le soir même la plupart du temps. ${ }^{59}$ »
\end{abstract}

Dans l'agglomération lyonnaise, les procès des meurtriers de Wahid Hachichi et d'Ahmed Bouteldja sont des cas emblématiques et médiatisés des tensions entre police, justice et jeunes de banlieue (ces cas sont médiatisés et connus des jeunes de banlieue). Hachichi est tué à Lyon le 18 octobre 1982 par le policier Nicolas Bartelli et Bouteldja le 28 septembre 1982 par un habitant de Bron, Jean-Claude Lopez, de deux balles dans le dos. Bartelli est laissé libre après son inculpation tandis que Lopez ne fait que six mois de détention provisoire. Leur mise en liberté - puis, après les événements de 1983, la relative clémence des verdicts (sept ans de prison ferme pour Bartelli et quatre pour Lopez) prononcés en 1985 par le juge et le jury de la Cour d'Assises de Lyon - est perçue par les familles et les amis de quartier, selon les termes d'Abdelmalek Sayad, comme « une manière de complicité objective avec l'assassin qui est leur compatriote, une marque de solidarité plus organique et qui n’a pas besoin de réfléchir pour exister 60 ». Dans ces cas de figure, la question de classe s'articule avec la question nationale-raciale : «on connait l'expression "justice de classe"; ici, il faudrait parler de "justice de caste", celle-ci venant doubler celle-là 61 ».

58. Boubeker A., Les mondes de l'ethnicité. La communauté d'expérience des héritiers de l'immigration maghrébine, Paris, Balland, 2003.

59. Entretien avec Toumi Djaïdja, Lyon, 11 décembre 2009.

60. Sayad A., "Exister, c'est exister politiquement. IIe partie : Les droits civiques pour une plus grande justice », Presse et Immigrés en France, 136, décembre 1985, p. 12. C’est moi qui souligne.

61. Ibid. 
L'enjeu de la solidarité étatique est double : celle-ci est non seulement dénoncée par les habitants des banlieues en raison de la perception d'une « impunité » policière, mais elle est aussi revendiquée par certaines composantes de l'État afin d'éviter de «ternir l'institution policière et d'en amenuiser la réputation, d'en altérer la face, au sens fort de l'expression goffmanienne 62 ». La volonté de maintenir les liens de la solidarité étatique conduit les individus ou organismes chargés de sanctionner les déviances policières, notamment l'Inspection générale de la police nationale (IGPN), à faire preuve d'une relative clémence concernant l'usage abusif de la violence physique. Autrement dit, le principe de solidarité étatique revient à ne pas faire " perdre la face à l'État » lorsque la police faillit, dans la mesure où la reconnaissance étatique des déviances policières violentes est considérée, à tort ou à raison, comme une perte de légitimité politique. Selon ce point de vue, la légitimité du monopole de l'usage de la violence physique est fondée sur un «bon » usage et elle s'effrite dès lors que l'État admet publiquement l'idée d'une forme institutionnelle (et non exceptionnelle) d'usages déviants.

Ainsi, l'équation « reconnaissance publique des déviances policières violentes $=$ discrédit et perte de légitimité de la police » est inscrite dans les catégories d'entendement des représentants des forces de l'ordre et elle est particulièrement bien révélée par les tenants et les aboutissants de l'« affaire Grasset ». Le 21 novembre 1983, en pleine Marche pour l'égalité (15 octobre 3 décembre), le jeune des Minguettes Abdelhamid Benatir (17 ans) est abattu par M. Lehouard d'une balle dans le dos. Ce meurtre a priori accidentel provoque de nouveaux troubles dans le quartier : les voitures des membres de la famille Lehouard sont incendiées et les forces de police établissent un périmètre de sécurité autour du domicile du tireur. Mais une compagnie de CRS sort de ce périmètre sous prétexte de poursuivre des individus "suspects " pénétrant dans le bar «L'Ami Burger » qui est, comme on l'a vu, un des lieux de vie de prédilection des jeunes des Minguettes.

Cette intervention brutale, réalisée par des CRS venus d'ailleurs et ne connaissant pas le quartier, bouleverse le fragile équilibre des tensions aux Minguettes, d'autant plus que le contexte de la Marche pour l'égalité focalise l'attention gouvernementale sur la situation locale. La brutalité est rapidement dénoncée non seulement par les habitants du quartier, mais aussi par le préfet de police : « [ils ont pénétré] de leur propre initiative dans ce bistro. [Il s’agissait] de jeunes CRS, jouant les gros bras, [qui] n'étaient pas habitués. Ils finissent par se faire chambrer par les habitués [du bar], s'affolent et se mettent quand même à tirer avec des balles qu'on retrouve à deux mètres de hauteur. Bon alors là-dessus, on rétablit l'ordre difficilement 63 ». Dans ce contexte tendu, Grasset condamne publiquement, devant les caméras de FR3, le comportement des CRS :

62. Moreau de Bellaing C., art. cit., p. 140.

63. Entretien avec Bernard Grasset, Rochefort, 22 février 2009. 
«Le commissaire principal de Vénissieux, Daniel Quantin, avait donné des ordres oraux et écrits à une patrouille pour assurer la garde autour du domicile du tireur pendant son interrogatoire à l'hôtel de police. Cette patrouille a élargi sans raison son périmètre. Elle est allée dans un bar du quartier où elle n'avait rien à faire. Elle a transgressé les ordres du commissaire. Ces policiers ont agi de leur propre initiative et c'est regrettable. Leur comportement dans ce bar a été inadmissible pour des fonctionnaires de police qui ne doivent pas se conduire comme les voyous qu'ils ont pourchassés ${ }^{64}$. »

Cette déclaration publique provoque un tollé général puisqu'il s'agit d'une rupture de la solidarité étatique de la part d'un préfet de police. Il déroge au principe de l' «inversion hiérarchique » selon lequel le rôle de la hiérarchie consiste non seulement à prescrire, mais aussi à justifier ou « rendre compatibles [les choix des « exécutants » du bas] avec les cadres réglementaires et les "politiques" définies par le haut 65 ». Il lui est reproché de ne pas «se converti[r] en porte-parole des intérêts corporatifs 66 » de son administration. Les syndicats de police considèrent ainsi qu'il est « inadmissible (...) qu'un préfet de police puisse se comporter de la sorte » parce que, «préjugeant le résultat des enquêtes administratives et judiciaires en cours, il vient de condamner les fonctionnaires dont il est, paraît-il, le chef... 67 ». Ils dénoncent un « lâchage éhonté » et l' « irresponsabilité totale d'un haut-fonctionnaire qui n'hésite pas, pour préserver sa carrière, à mettre en péril celle de ses subordonnés ", ce qui les conduit à « exiger » le départ de ce préfet de police « qu'ils ne reconnaissent plus comme l'un des leurs». La section lyonnaise de Force ouvrière Police condamne "la rapidité d'une telle prise de position [qui] ne peut que discréditer l'ensemble du corps de la police nationale ». Pour Roger Guichard (élu du Rassemblement pour la République), il est «néfaste qu’un chef de la police désavoue ses hommes » et pour Gaston Deferre, ministre de l'Intérieur socialiste, "le gouvernement est derrière sa police ». Yvon Deschamps, secrétaire fédéral du Parti socialiste, est « embarrassé » et affirme que « le préfet a agi en politique plus qu'en haut fonctionnaire. Il a voulu faire tomber la tension aux Minguettes, ce qui était normal, mais peut-être aurait-il dû faire ses déclarations au conditionnel, laissant seulement entendre qu'il désavouerait les policiers si l'enquête concluait qu'il y a eu faute de leur part ».

64. Cité par Leridon Y., "Les Minguettes : les incroyables propos du préfet », Le Figaro, 25 novembre 1983.

65. Montjardet D., Ce que fait..., op. cit., p. 89.

66. Monjardet D., "L'insécurité politique : police et sécurité dans l'arène électorale ", Sociologie du travail, 44-4, 2002, p.1.

67. Communiqué de l'intersyndicale Syndicat des corps en civil de la police nationale CGC, Syndicat des commandants et officiers de la police nationale CGC, Syndicat des gradés de la police nationale CGSC et Syndicat indépendant de la police nationale, publié dans $L e$ Progrès, 25 novembre 1983. Les citations suivantes de ce paragraphe sont extraites du même numéro de journal. 
Ainsi, les syndicats de police, le ministre de l'Intérieur et les principaux partis politiques condamnent les propos de Grasset qui s'est désolidarisé de ses troupes. Il n'est plus « un des leurs 68 ». Le préfet du Rhône, Olivier Philip, ne prend pas position publiquement et seules quelques personnalités, notamment de rares élus régionaux socialistes, le maire de Vénissieux Marcel Houël 69 et le député UDF et ancien ministre Raymond Barre, le soutiennent publiquement ou en privé 70 . Implicitement, le préfet de police avait adopté une stratégie inhabituelle : pour "faire tomber la pression ", il ne s'agit pas d'éviter de "perdre la face » et d'élever le niveau de répression, mais de reconnaître les déviances policières et l'usage abusif de la force physique. Autrement dit, cette reconnaissance publique n'est plus synonyme de discrédit et de perte d'autorité mais au contraire de gain de légitimité et de crédibilité vis-à-vis de la population. Cette inversion de l'équation reconnaissance/légitimité est une revendication explicite des jeunes des Minguettes :

«C'était à cette époque une culture de la gâchette facile. On tirait très facilement... [Les policiers savaient] qu'ils pouvaient faire justice eux-mêmes et qu'ils allaient être couverts par la loi. À un tel point que quand l'AFP et les journaux (...) ont diffusé l'information [un policier tire sur un jeune des Minguettes], c'était dans l'idée de calmer le jeu [et] de dire : "il faut calmer pour ne pas que la banlieue 's'enflamme'”. Mais ce n'est pas ce que la banlieue vous demande. La banlieue vous demande d'être juste, de reconnaitre les faits, de dire : "on a fait une bavure" ${ }^{71}$. "

Ainsi, «les marques de reproche ou d'hostilité visent moins, à cet égard, la violence comme catégorie générale de l'action policière que certaines de ses formes de violence, ou son intensité 72 ». Mais l'éphémère crédibilité retrouvée par la police auprès des habitants des Minguettes suite aux propos de Grasset s'articule avec une perte de légitimité auprès des agents de police qui se sentent trahis, comme ils s'étaient sentis trahis par la suspension des expulsions des étrangers condamnés par le gouvernement socialiste en 1981. C’est pour « combler la brèche » dans la solidarité étatique et rétablir la confiance des troupes que le préfet du Rhône, Olivier Philip ${ }^{73}$, intervient auprès d'une

68. Ibid.

69. Le Progrès, 26 novembre 1983.

70. Entretien avec Bernard Grasset, Rochefort, 22 février 2009.

71. Entretien avec Toumi Djaïjja, Lyon, 25 décembre 2009.

72. Jobard F., Bavures..., op. cit., p. 66.

73. Olivier Philip (1925-) est issu d'une célèbre famille protestante lyonnaise. Il est le fils du ministre, député (SFIO) du Rhône et professeur de droit André Philip (1902-1970). Docteur en droit, il est diplômé de l'École spéciale militaire de Saint-Cyr, ancien élève de l'École libre des sciences politiques et de l'École nationale d'administration (promotion France combattante en 1947). Il peut être qualifié de haut fonctionnaire gaulliste (il rejoint les Forces françaises libres de De Gaulle en 1941 et le cabinet du Premier ministre Georges Pompidou en 1966-1967) et devient préfet dans plusieurs départements (Guadeloupe, Limousin, Bretagne, 
soixantaine de policiers du commissariat de Vénissieux (document 1 ci-dessous). Son discours relève d'un discours privé et n'est pas contraint par les effets de censure propres au discours public. Le préfet révèle ainsi la « doctrine officieuse » de la police en cas de déviances policières : la hiérarchie « a toujours couvert publiquement » la « petite erreur » « quitte à ce qu'une sanction interne à la "maison" intervienne après enquête et sans publicité excessive ". Cependant, en cas de "grosse erreur» relevant surtout de la désobéissance aux ordres, "nous perdrons notre crédibilité si nous n'avons pas le courage de reconnaître les faits et les erreurs " et "si nous mentons». Autrement dit, la solidarité étatique ne peut plus être maintenue dès lors qu'elle compromet la crédibilité et la légitimité de la police auprès de la population.

Cependant, la condamnation par un préfet de police des déviances policières est particulièrement rare. La distinction entre «petite » et « grosse » erreur n'est pas une donnée évidente mais bien au contraire un enjeu de lutte entre les différents acteurs de la solidarité étatique, qui ne se sont pas tous d'accord entre eux. Ainsi, l'enquête diligentée par l'IGPN requalifie les déviances policières en question en «petite » erreur, dans la mesure où les CRS incriminés n’auraient pas « dérogé exagérément aux pratiques policières en milieu dangereux ou hostile » et ne méritent donc pas de «peu souhaitables sanctions » (document 2). La brèche entrouverte en public par Grasset est immédiatement refermée par l'IGPN sans publicité, ce qui permet de faire « retomber la pression » du côté des forces de police.

\section{Document 1 : La " doctrine officieuse de la bavure policière ".}

Discours du préfet Olivier Philip devant les policiers du commissariat de Vénissieux, 30 novembre 1983.

«Excluant l'hypothèse de la grosse erreur (...), votre hiérarchie a toujours couvert publiquement ce qu'on l'on peut appeler la petite erreur. On peut commettre une petite erreur dans le feu de l'action. Vous devez être couvert, quitte à ce qu'une sanction interne à la "maison" intervienne après enquête et sans publicité excessive. L'incident de la semaine dernière est une "grosse erreur" sur deux points :

a) erreur en elle-même. Vous savez ce qui s'est passé. Je n'insisterai pas, notamment sur l'attitude verbale adoptée, sur le fait que les locaux ont été saccagés et pas seulement semble-t-il par des clients, sur le fait que l'on a obligé les jeunes à se coucher par terre sous la menace. Une balle a été tirée à hauteur d'homme et la tragédie évitée de justesse. L'Inspection Générale vérifie les détails et en tirera les conclusions qu'elle jugera utile.

b) erreur par la désobéissance aux ordres. C'est sans doute l'aspect le plus grave de cette affaire. La mission donnée aux CRS, consignée par écrit par le Commissaire responsable, l'a été dans le cadre d'un contexte qu'il connaissait. (...) [L]'ordre a été donné d'intervenir pour protéger la famille française de l'auteur de l'homicide (accidentel ou non), et dont deux voitures venaient d'être incendiées. Telle était la mission. (...) Or, 4 CRS sont allés effectuer un contrôle d'identité dans un café situé à 800 mètres du lieu d'intervention. Tout s'est passé à peu près normalement, mais ils y sont retournés peu

etc.). Il est préfet de la région Rhône-Alpes et du département du Rhône entre 1978 et septembre 1984, préfet de la région Île-de-France et du département de Paris entre septembre 1984 et janvier 1991. 
après. Pour ce faire, ils ont abandonné leur mission. (...) Un Chef ne peut pas être désobéi. Le Chef doit supporter les conséquences des ordres qu'il donne et il doit les assumer. Mais vous devez comprendre qu'un responsable n'a pas à supporter, n'a pas à assumer les conséquences d'un acte lorsqu'on lui a désobéi.

Les faits étaient évidents, immédiatement connus de la Presse, la désobéissance flagrante. La Télévision régionale étant saisie et plusieurs européens du quartier y intervenant pour relater les faits, il était indispensable pour le Préfet délégué pour la Police d'intervenir publiquement. C'est ce qu'il a fait à ma demande. II n'était pas possible d'attendre car il faut réagir immédiatement à l'événement dans ce genre de situation. C'est l'intérêt de la Police. Celle-ci doit être crédible aux yeux de la population. Nous perdrons notre crédibilité si nous n'avons pas le courage de reconnaître les faits et les erreurs. Nous perdrons la confiance de la population si nous mentons. Vous savez bien que la vérité est vite connue dans le quartier. Vous êtes susceptibles d'intervenir à tout moment aux Minguettes pour assurer dans des conditions difficiles l'ordre public. La population doit avoir confiance en votre impartialité. Nous devons êtres crédibles, non seulement vis-à-vis de la population mais aussi vis-à-vis du Gouvernement. ${ }^{74}$ »

\section{Document 2 : Rapport d'enquête de l'Inspection générale de la police nationale relatif à l'incident du 21 novembre 1983 aux Minguettes.}

«Le rapport rassemblé (...) apporte un certain nombre d'éléments pouvant donner un éclairage assez complet sur cette affaire dont le déroulement est surtout marqué par l'influence prismatique de son environnement.

S'il n'y avait eu auparavant mort vraisemblablement accidentelle d'un jeune homme. S'il n'y avait eu un conflit ouvert entre une communauté maghrébine sensibilisée à l'extrême par son deuil et une famille européenne menacée dans sa sécurité. S'il n'y avait eu le climat particulier propre à la Cité des Minguettes où tout ce qui touche aux relations police-population prend une résonance quasi-nationale. S'il n'y avait eu tout ce contexte passionnel encore plus accusé par les relations des médias, il resterait une banale affaire de drogue pour laquelle on eut sans doute félicité les jeunes fonctionnaires pour leur zèle en les rappelant néanmoins à un peu plus de mesure, y compris vis-à-vis des repris de justice lors de leur interpellation. Une fermeture administrative aurait sanctionné l'établissement fautif $\mathrm{d}^{\prime}$ accueillir des trafiquants de ce pernicieux et alarmant trafic.

Mais il s'agit précisément des Minguettes. Alors, il en va différemment. On se renvoie les responsabilités, car on redoute les conséquences de cette intempestive intervention dont la forme a excédé les principes du droit, sans déroger exagérément aux pratiques policières en milieu dangereux ou hostile. (...)

S'agissant des mesures à prendre, (...) il faut rappeler à la hiérarchie des CRS que les contacts avec les services renforcés doivent être permanents (...). En toute hypothèse, peut-on demander, afin d'éviter tout incident, à des fonctionnaires de police de fermer les yeux sur un délit aussi grave que le trafic de drogue alors qu'on les incite, par ailleurs fort pertinemment, à lutter contre ce fléau ? C'est sans doute à cette dernière question qu'il convient de répondre avant de se prononcer sur de peu souhaitables sanctions. 75 »

74. ADR 1858W1. Discours du préfet Olivier Philip devant une soixantaine de policiers dépendant du commissariat de Vénissieux, 30 novembre 1983.

75. Archives de l'IGPN, sans cote. «Enquête sur les circonstances d'un incident survenu le 21 novembre 1983 lors d'une intervention de police dans la Cité des Minguettes », 7 décembre 1983. 
Pour conclure, l'exemple des rébellions des Minguettes permet de mettre en lumière les conditions de possibilité de la contestation de l'autorité policière par les gouvernés. La probabilité de rébellion doit moins au décalage entre attentes et satisfactions, cher à la théorie de la frustration relative, mais beaucoup aux données fondamentales de la configuration locale, et notamment à la rupture de l'équilibre des tensions dans les relations entre la police et les jeunes de banlieue. Si on admet cette idée, la comparaison entre plusieurs rébellions urbaines suppose une attention particulière aux acteurs et rapports de force locaux. Par ailleurs, la rébellion de 1983 est un exemple plutôt rare de conversion des émeutiers au répertoire de l'action collective, rendue possible par l'action d'habitants politisés aux Minguettes et la prise de conscience d'un rapport de forces défavorable.

De plus, l'analyse d'entretiens et d'archives inédits met en lumière les non-dits des tensions entre jeunes de banlieue et forces de police. Les premiers dénoncent l'« impunité » policière et demandent la reconnaissance des déviances policières dans l'usage de la force physique. La tension est d'autant plus forte que les jeunes perçoivent un degré élevé de solidarité étatique, ce qui participe, au niveau structurel, à la perpétuation du sentiment d'injustice et, au niveau conjoncturel, à la perpétuation et à l'extension géographique de la rébellion. Mais, de manière générale et au sein même des forces de police, s'opposent deux conceptions diamétralement opposées du rapport légitimité/reconnaissance. D’un côté, la reconnaissance publique des déviances policières violentes est considérée comme un des fondements de la légitimité alors que, de l'autre, cette reconnaissance publique mine la solidarité d'un appareil étatique qui doit se « serrer les coudes» et ne pas « perdre la face ». 